\title{
Influência de fatores antropométricos e atividade física na pressão arterial de adolescentes de Taguatinga, Distrito Federal, Brasil
}

\author{
Influence of anthropometric factors and physical activity on blood \\ pressure in adolescents from Taguatinga, Federal District, Brazil
}

\author{
S.L. Silva, B. Madrid, C.M. Martins, J.L. Queiroz, M.T. Dutra, F.M. Silva
}

ARTIGO ORIGINAL | ORIGINAL ARTICLE

\begin{abstract}
RESUMO
O estudo objetivou analisar a influência do índice de massa corporal (IMC) e do nível de atividade física (NAF) sobre a pressão arterial (PA) em adolescentes. A amostra foi constituída por 799 estudantes de ambos os sexos, com idade média de 12.09 anos ( \pm 0.80 ). O peso, a estatura e a PA foram avaliados de acordo com critérios internacionalmente estabelecidos. Em seguida, procedeu-se ao cálculo do IMC e os sujeitos foram classificados de acordo com os pontos de corte propostos por Cole, Flegal e Dietz (2000). Para avaliar o NAF, procedeu-se ao preenchimento do questionário de estilo de vida modificado (Silva, Silva, \& Martins, 2006). Observou-se que 9.1\% dos adolescentes eram hipertensos, $11.8 \%$ tinham sobrepeso, $5.4 \%$ eram obesos e $47.2 \%$ dos sujeitos apresentaram baixo NAF. O fato de ser inativo (OR $=1.99)$, insuficientemente ativo $(\mathrm{OR}=1.87)$ e ser obeso $(\mathrm{OR}=5.1)$ apresentou associação com o aumento das chances de desenvolver hipertensão. Ser inativo e insuficientemente ativo está fortemente associado ao desenvolvimento da obesidade ( $\mathrm{OR}=7.97$ e 4.35 ) respectivamente. $\mathrm{Na}$ amostra estudada, o sobrepeso, a obesidade e o baixo NAF estão associados ao desenvolvimento de hipertensão arterial. Além disso, um baixo NAF está associado ao aumento de peso nos adolescentes.

Palavras-chave: índice de massa corporal, nível de atividade física, pressão arterial, adolescentes
\end{abstract}

ABSTRACT

This study aimed to analyze the influence of body mass index (BMI) and physical activity level (PAL) on blood pressure (BP) in adolescents. The sample was composed of 799 students of both genders, aged 12.09 ( \pm 0.80). The weight, height and BP were assessed according to internationally established criteria, and BMI was calculated and classified according to the cutoff points proposed by Cole, Flegal and Dietz (2000). To evaluate the PAL, the modified lifestyle questionnaire was used (Silva, Silva, \& Martins, 2006). We found that $9.1 \%$ of the subjects evaluated have high blood pressure, $11.8 \%$ were overweight, $5.4 \%$ were obese and $47.2 \%$ of the people presented low PAL. The fact of being inactive $(\mathrm{OR}=1.99)$, insufficiently active $(\mathrm{OR}=1.87)$ and obese $(\mathrm{OR}=5.1)$ was associated with an increased risk of developing hypertension. Being inactive or insufficiently active was strongly associated with the development of the obesity ( $\mathrm{OR}=7.97$ and 4.35$)$ respectively. In the studied sample, the overweight, the obesity and low PAL are associated with the development of arterial hypertension. In addition, a low PAL is associated with an increasing number of overweight adolescents.

Keywords: body mass index, physical activity level, blood pressure, students

Submetido: 25.06.2012 | Aceite: 13.01.2013

Sebastião Lobo Silva, Bibiano Madrid. Faculdade Albert Einstein, Brasília, Brasil.

Clarice Maria Martins. Instituto Superior da Maia, Porto, Portugal.

José Luiz Queiroz, Francisco Martins Silva. Universidade Católica de Brasília, Brasil.

Maurilio Tiradentes Dutra. Universidade de Brasília, Brasil.

Endereço para correspondência: Maurílio Tiradentes Dutra, Rodovia BR 020 Km 03 Condomínio Granville, № 25,

Região dos Lagos - Sobradinho, Distrito Federal, CEP: 73251-904, Brasil.

E-mail:mtd71@excite.com 
Os avanços tecnológicos ocorridos nos últimos anos contribuíram para modificar os hábitos de vida de grande parte da população, incluindo os adolescentes. Esses avanços trouxeram vários benefícios, mas favorecem a inatividade física e o aumento do peso corporal, contribuindo para o surgimento de doenças crônicas não transmissíveis, dentre elas a hipertensão arterial (HA) e a obesidade (Brasil, Fisberg, \& Maranhão, 2007).

Nesse sentido, a Organização Mundial de Saúde (OMS) relatou que no ano de 2004 existia aproximadamente 1 bilhão de pessoas com sobrepeso, destes, 300 milhões eram consideradas obesas de acordo com o nível de classificação de índice de massa corporal (IMC $>30 \mathrm{~kg} / \mathrm{m}^{2}$ ). No Brasil, $43 \%$ da população está com excesso de peso corporal e, em Brasília, $13 \%$ dos adolescentes estão nessa mesma situação (Brasil, 2004), enquanto no Rio de Janeiro $12.5 \%$ dos adolescentes avaliados estavam acima do peso considerado normal (Chiara, Sichieri, \& Martins, 2003). Em relação aos adolescentes americanos, $16 \%$ deles encontram-se com excesso de peso (Hedley et al., 2004).

O baixo nível de atividade física (NAF) continua sendo fator determinante para $O$ desenvolvimento de doenças crônicas não transmissíveis (Brasil et al., 2007; Guerra, Ribeiro, Duarte, \& Mota, 2003; World Health Organization [WHO], 2003). Sendo que adolescentes do mundo todo possuem índices de sedentarismo ou inatividade física variando entre 51 e $60 \%$ (Araújo et al., 2008; Farias \& Salvador, 2005; Pierine et al., 2006).

A HA é considerada uma doença incomum em adolescentes, porém sua incidência vem aumentando significativamente nos últimos anos. A HA é considerada um fator primordial de risco para o desenvolvimento de outras doenças. Por ser considerada uma patologia de alta morbidade, o diagnóstico precoce é importante para o tratamento e prevenção nas primeiras etapas da vida (National High
Blood Pressure Education Program Working Group on High Blood Pressure in Children and Adolescents, 2004). Atualmente, $26.4 \%$ da população mundial é considerada hipertensa e este quadro tende a se tornar ainda mais grave nos próximos anos, com um aumento estimado para 29\% no ano de 2025 (Kearney et al., 2005). Em crianças e adolescentes, a HA varia de 2.7 a 19.4\% (Monge \& Beita, 2000; Sorof, Lai, Turner, Poffenbarger, \& Portman, 2004). Especificamente no Brasil, a incidência de HA em adolescentes varia de 3.1 a $13.3 \%$ (Moura, Silva, Ferraz, \& Rivera, 2004; Oliveira et al., 2004; Romanzini, 2006).

O baixo NAF contribui para o aumento do peso corporal e este pode contribuir para $\mathrm{O}$ surgimento da HA. Em estudos internacionais, foi encontrada relação entre o IMC, NAF e a pressão arterial (PA) (Sorof et al., 2004). Então, faz-se necessário um diagnóstico precoce para evitar os riscos causados pela HA. Contudo, não foram encontrados estudos investigando a associação destas variáveis em adolescentes na região do Distrito Federal, Brasil. Portanto, o objetivo deste estudo foi analisar os níveis de associação do IMC e do NAF com a PA em adolescentes brasileiros de 11 a 13 anos, da cidade de Taguatinga, Distrito Federal, Brasil.

\section{MÉTODO}

O design experimental caracterizou-se como epidemiológico, de corte transversal. O estudo foi aprovado pelo Comitê de Ética em Pesquisa com Seres Humanos da Universidade Católica de Brasília (034/2007).

\section{Amostra}

A amostra foi composta por adolescentes, de ambos os sexos, com idade entre 11 e 13 anos (12.09 \pm 0.8 anos), estudantes de escolas públicas e privadas de Taguatinga, cidade-satélite do Distrito Federal, Brasil. Para selecionar as escolas que fizeram parte da amostra, a cidade foi dividida geograficamente em três regiões: norte, centro e sul. De cada região, foram selecionadas de forma intencional as duas 
maiores escolas (uma pública e uma privada). Nestas escolas foi distribuído aos estudantes de 11 a 13 anos o termo de consentimento livre e esclarecido, a fim de ser assinado pelos pais ou responsáveis.

O tamanho da amostra foi calculado a partir da proposta de Luiz e Magnanini (2000), para estudos epidemiológicos considerando o intervalo de confiança de $95 \%$, erro tolerável de $3 \%$ e uma prevalência estimada de adolescentes hipertensos em torno de 10\% (Oliveira, Lamounier, Oliveira, Castro, \& Oliveira, 1999). Definiu-se um efeito de delineamento igual a 1.9 e estimou-se o tamanho da amostra mínima de 633 adolescentes e, por segurança, decidiu-se acrescentar $30 \%$ para evitar eventuais perdas ocasionadas por recusa a participar do estudo ou preenchimento incorreto do questionário de NAF, totalizando-se numa amostra possível e significativa de 823 estudantes, escolhidos de forma aleatória de um universo de 2464 alunos nas escolas escolhidas para o estudo. Portanto, os estudantes que não atenderam aos critérios estabelecidos, não assinaram o termo de consentimento livre e esclarecido, faltaram a alguma fase da coleta e estavam tomando algum medicamento anti-hipertensivo foram excluídos do estudo, resultando em uma amostra final de 799 adolescentes.

\section{Instrumentos}

A medida antropométrica seguiu normas padronizadas por Lohman, Roche, e Martorell (1988). Para aferir a massa corporal $(\mathrm{kg})$, utilizou-se uma balança digital da marca Plenna $^{\circledR}$ e a estatura $(\mathrm{cm})$ foi aferida com um estadiômetro da marca Seca ${ }^{\circledR}$. O NAF dos adolescentes foi identificado e classificado através do questionário de estilo de vida de Barros e Nahas (2003), modificado por Silva, Silva, e Martins (2006), que mede um dia típico de atividades físicas. As aferições da PA foram realizadas por um único avaliador pelo método auscultatório, mediante a utilização de um esfigmomanômetro aneroide infantil, devida- mente calibrado.

\section{Procedimentos}

Os adolescentes foram classificados quanto ao IMC/idade por meio dos pontos de corte estabelecidos por Cole, Bellizzi, Flegal, e Dietz (2000).

O questionário de estilo de vida foi aplicado em sala de aula por três avaliadores previamente treinados, e os estudantes responderam perguntas sobre os tipos de atividades normalmente realizadas no dia a dia com destaque para os esportes com bola, ciclismo, dança, atividades recreativas (bola de gude, barra bandeira, baleado, entre outras), domésticas (espanar pó, jogar lixo, lavar louças e roupas) e formas de deslocamentos da residência para a escola (bicicleta, a pé, carro e outros). Para a conversão das informações obtidas na AF em valores estimados de dispêndio energético, recorreu-se ao compêndio de AF que oferece informações sobre o gasto energético em unidades do equivalente metabólico de trabalho para uma atividade particular (MET), com quase quinhentas formas de atividades físicas (Ainsworth et al., 2000). Em seguida, estabeleceram-se os pontos de corte por meio do escore geral, obtido na soma das AF, utilizando a classificação de quartis, sendo consideradas inativas fisicamente as crianças com escore abaixo do percentil 25; insuficientemente ativas percentil entre 25 e 50 , moderadamente ativas percentil entre 50 e 75 e ativas para valores acima do percentil 75 .

As aferições da PA seguiram as orientações sugeridas pela IV Diretrizes Brasileiras de Hipertensão Arterial (Sociedade Brasileira de Cardiologia [SBC], Sociedade Brasileira de Hipertensão [SBH] e Sociedade Brasileira de Nefrologia [SBN], 2004), tendo atenção de que nos 30 minutos precedentes ao início das aferições, os adolescentes não praticaram exercícios físicos, ingeriram café ou outros tipos de alimentos. Além disso, os adolescentes foram orientados a urinar antes do início das aferições, 
a permanecerem por no mínimo cinco minutos sentados e em repouso. Duas medidas foram realizadas para cada adolescente, sendo que, caso ocorresse uma diferença igual ou maior a quatro milímetros de mercúrio $(\mathrm{mmHg})$ entre as aferições, uma terceira mensuração seria realizada. Portanto, para fins de análise, considerou-se o valor médio da PAS e PAD referentes às duas medidas ou o valor médio de três aferições, quando necessário. Os adolescentes foram considerados hipertensos quando seus valores da pressão arterial diastólica ou sistólica estivessem acima do percentil 95, de acordo com sua idade, sexo e estatura (National High Blood Pressure Education Program Working Group on High Blood Pressure in Children and Adolescents, 2004).

\section{Análise Estatística}

Analisou-se a normalidade dos dados por meio do teste de Kolmogorov Smirnov. O teste Qui-quadrado $\left(x^{2}\right)$ possibilitou verificar as diferenças significativas entre as classificações do NAF e IMC em relação à classificação da PA. A análise de regressão logística univariada foi empregada para verificar as razões de chances existentes entre NAF, IMC e PA e também entre o IMC e o NAF especificamente. O nível de significância adotado foi de $p \leq .05$. O programa utilizado foi o SPSS (versão 11.0 para Windows) e todos os testes estatísticos e fórmulas estão de acordo com as instruções de Field (2009).

\section{RESULTADOS}

A tabela 1 apresenta os dados de caracterização da amostra estratificada somente em sexo masculino e feminino.

A tabela 2 apresenta a análise descritiva da amostra, com indicações da frequência absoluta e percentual dos adolescentes nas variáveis IMC, NAF e PA. Os estudantes avaliados possuíam médias de idade de $12.08( \pm 0.81)$ e $12.10( \pm 0.80)$ anos para os sexos masculino e feminino, respectivamente. Observou-se que
$11.8 \%$ da amostra eram sobrepesados e $5.4 \%$ obesos. Em relação à AF, $47.2 \%$ apresentaram baixo NAF (22.3\% inativos e $24.9 \%$ insuficientemente ativos). Adicionalmente, $9.1 \%$ dos sujeitos avaliados foram classificados como hipertensos. Quanto ao NAF, os grupos apresentaram número de indivíduos semelhantes em termos percentuais. Ao comparar as categorias de PA e de IMC, foram verificadas diferenças significativas entre os indivíduos normotensos e eutróficos $(p=.01)$, em relação aos normotensos e sobrepesados ou obesos. Vale ressaltar que, apesar de não significativa em relação às demais categorias, nos adolescentes classificados com obesidade há uma tendência à HA.

A tabela 3 apresenta os resultados da associação entre a $\mathrm{HA}$ e as categorias do NAF e do IMC. Observou-se que a chance de desenvolver HA foi quase duas vezes maior, tanto nos adolescentes considerados inativos quanto nos insuficientemente ativos $(p=.01 ; p=.03)$. Assim, ao considerar a totalidade de adolescentes com baixos NAF (inativos e insuficientemente ativos), a chance de desenvolver HA aumentará quase quatro vezes em relação aos adolescentes ativos. Ao avaliar adolescentes moderadamente ativos e ativos, a associação não foi significativa. Portanto, observa-se que quanto menor o nível de atividade física maior a chance de desenvolver HA.

$\mathrm{Na}$ avaliação do grau de associação do IMC com HA, observou-se que a chance de desenvolver HA foi duas vezes maior em adolescentes sobrepesados $(p=.14)$ e cinco vezes maior em adolescentes obesos $(p=.02)$ em comparação com os eutróficos.

A tabela 4 apresenta a associação entre obesidade e diferentes categorias de NAF. Os resultados indicaram que adolescentes inativos apresentaram chance quase oito vezes maior de terem excesso de peso, enquanto que para os insuficientemente ativos, as chances aumentaram em cerca de quatro vezes. Assim, os dados indicam que na amostra estudada, há 
uma forte relação entre ter baixos NAF (inativo ou insuficientemente ativo) e o desenvolvimento e excesso de peso, fato este que não se verifica para adolescentes moderadamente ativos ou ativos.

Tabela 1

Caracterização da amostra estratificada somente em sexo masculino e feminino

\begin{tabular}{cccc}
\hline \multirow{2}{*}{ Variáveis } & \multicolumn{2}{c}{ Sexo } & \multirow{2}{*}{ TOTAL $(n=799)$} \\
\cline { 2 - 3 } & Masculino $(n=368)$ & Feminino $(n=431)$ & $12.09 \pm 0.80$ \\
IDADE (anos) & $12.07 \pm 0.81$ & $12.10 \pm 0.80$ & $18.20 \pm 2.87$ \\
IMC & $18.09 \pm 3.0$ & $18.29 \pm 2.75$ & $132.71 \pm 55.33$ \\
NAF & $144.77 \pm 54.33$ & $122.33 \pm 54.12$ & $104 \pm 17$ \\
PAS & $104 \pm 15$ & $103 \pm 20$ & $66 \pm 9$ \\
PAD & $66 \pm 9$ & $65 \pm 10$ & \\
\hline
\end{tabular}

Nota: NAF: nível de atividade física. IMC: índice de massa corporal em $\mathrm{Kg} / \mathrm{m}^{2}$. PAS: pressão arterial sistólica em mmHg. PAD: pressão arterial diastólica em mmHg.

Tabela 2

Características descritivas da amostra por gênero

\begin{tabular}{ccccc}
\hline \multirow{2}{*}{ Variáveis } & & \multicolumn{3}{c}{ Sexo } \\
& & \multicolumn{4}{c}{ Feminino } \\
& & $(46.1 \%)$ & $(53.9 \%)$ & \\
\cline { 2 - 4 } & Eutrófico & $(307) 38.4 \%$ & $(355) 44.4 \%$ & $(662) 82.9 \%$ \\
IMC & Sobrepesado & $(35) 4.4 \%$ & $(59) 7.4 \%$ & $(94) 11.8 \%$ \\
& Obeso & $(26) 3.3 \%$ & $(17) 2.7 \%$ & $(43) 5.4 \%$ \\
\cline { 2 - 5 } & Inativo & $(72) 19.6 \%$ & $(106) 24.6 \%$ & $(178) 22.3 \%$ \\
& Ins. Ativo & $(87) 23.6 \%$ & $(112) 26.0 \%$ & $(199) 24.9 \%$ \\
& Mod. Ativo & $(90) 24.5 \%$ & $(122) 28.3 \%$ & $(212) 26.5 \%$ \\
& Ativo & $(119) 32.3 \%$ & $(91) 21.1 \%$ & $(210) 26.3 \%$ \\
\cline { 2 - 5 } & Normotensos & $(339) 46.7 \%$ & $(387) 53.3 \%$ & $(726) 90.9 \%$ \\
& Hipertensos & $(29) 39.7 \%$ & $(44) 60.3 \%$ & $(73) 9.1 \%$ \\
\hline
\end{tabular}

Nota: IMC: índice de massa corporal. NAF: nível de atividade física. PA: pressão arterial.

Tabela 3

Associação entre HA e categorias de NAF e IMC

\begin{tabular}{ccccc}
\hline \multirow{2}{*}{ Variáveis } & Categorias & OR & IC $(95 \%)$ & $p$ \\
\hline \multirow{3}{*}{ NAF } & Inativo & 1.99 & $(1.17 ; 3.23)$ & $.01^{*}$ \\
& Insuficientemente Ativo & 1.87 & $(1.06 ; 2.46)$ & $.03^{*}$ \\
& Moderadamente Ativo & 0.93 & $(0.53 ; 1.51)$ & .08 \\
\cline { 2 - 5 } IMC & Ativo & 1.0 & - & - \\
& Eutrófico & 1.0 & - & $.001^{*}$ \\
\hline
\end{tabular}

Nota: NAF: nível de atividade física. IMC: índice de massa corporal. OR: oddsratio. IC: intervalo de confiança. P: nível de significância. ${ }^{*} p \leq .05$; Categorias de referencia: ativos e eutróficos.

Tabela 4

Associação entre obesidade e categorias de NAF

\begin{tabular}{ccccc}
\hline \multirow{2}{*}{ Variável } & Categorias & OR & IC $(95 \%)$ & $p$ \\
\hline \multirow{3}{*}{ NAF } & Inativo & 7.97 & $(3.66 ; 15.19)$ & $.001^{*}$ \\
& Insuficientemente ativo & 4.35 & $(2.79 ; 8.21)$ & $.02^{*}$ \\
& Moderadamente ativo & 1.97 & $(0.93 ; 1.53)$ & .13 \\
\hline
\end{tabular}

Nota: NAF: nível de atividade física. OR: odds ratio. IC: intervalo de confiança. P: nível de significância. ${ }^{*} p \leq$ .05 . 


\section{DISCUSSÃO}

O presente estudo teve como objetivo analisar a influência do IMC e do NAF sobre a PA de adolescentes de ambos os sexos da cidade de Taguatinga. Após análise dos resultados, observou-se que $9.1 \%$ dos adolescentes analisados são portadores de HA. Essa proporção é inferior àquela observada em pesquisas envolvendo jovens americanos (19.4\%), canadenses $17.0 \%$ e belgas $11.4 \%$ (Paradis et al., 2004; Paulus, Saint-Remy, \& Jeanjean, 1999; Sorof et al., 2004). No entanto, superior à encontrada em adolescentes costarriquenhos e portugueses: $2.7 \%$ (Monge \& Beita, 2000) e 5.9\% (Guerra et al., 2003), respectivamente.

Ao considerar o quadro nacional brasileiro, o presente estudo apresenta valores de HA de adolescentes inferiores aos estudos realizados nas capitais Maceió e Florianópolis, onde foram encontrados valores de $13.3 \%$ (Moura et al., 2004) e $12.0 \%$ (Giugliano \& Melo, 2004), respectivamente, e superior aos valores encontrados em Belém, 3.8\% (Jardim et al., 2011), Feira de Santana, 3.6\% (Oliveira et al., 2004) e Florianópolis, 3.1\% (Romanzini, 2006). Tanto em estudos brasileiros quanto internacionais, a presença de hipertensão em adolescentes é uma realidade preocupante. Vale destacar ainda que, apesar de inferiores, ao comparar com outros estudos desenvolvidos na Europa e América do Norte, ou até mesmo em outras cidades brasileiras, esses dados tomam maior proporção ao considerar que adolescentes hipertensos têm chances acrescidas de se tornarem adultos hipertensos (Sorof et al., 2004).

No Brasil, confirmando uma tendência de um cenário mundial, a população está progressivamente mais inativa (Associação Brasileira para o Estudo da Obesidade e Síndrome Metabólica [ABESO], 2008). Essa verdade também foi reforçada para a população em estudo, ao se verificar que os adolescentes de Taguatinga-DF, Brasil, apresentaram baixos NAF (47.2\%).
Resultados ainda mais agravantes do que os do presente estudo foram encontrados em Porto Velho (54\%), Botucatu (60\%) e em Pelotas (54.9\%), onde mais de metade da população foi considerada com níveis inferiores aos recomendados de AF (Azevedo, Araújo, Silva, \& Hallal, 2007; Farias \& Salvador, 2005; Pierine et al., 2006). Os adolescentes de Taguatinga-DF, Brasil, apesar de apresentarem NAF ligeiramente superiores ao comparar com os acima citados, não apresentam valores de NAF recomendados para uma população jovem, podendo ser um potencial fator o surgimento e desenvolvimento de doenças crônicas não transmissíveis.

Os resultados demonstrados na tabela 2 ressaltam que as chances de desenvolver HA foram quase duas vezes maiores à medida que os NAF fossem diminuindo, mais especificamente para as categorias "inativos" e "insuficientemente ativos". Já as categorias "moderadamente ativos" e "ativos" não apresentaram chances significativas em relação ao aumento da PA. Porém, estudos têm relatado divergências de opiniões no que se refere às associações entre AF e PA. O estudo de Pileggi, Carvone, Nobile, e Pavia (2005), envolvendo adolescentes italianos não identificou associações entre a prática de AF e a prevalência de HA. No Brasil, Romanzini (2006) em Florianópolis e Silva \& Farias Júnior (2007) em João Pessoa, também verificaram falta de relação entre NAF e PA em adolescentes. Contrapondo tais resultados, Guedes, Guedes, Barbosa, e Oliveira (2006) verificaram em adolescentes de Londrina uma associação significativa entre AF e PA, ao evidenciarem que adolescentes com baixo NAF apresentaram 80 e $91 \%$ de chances acrescidas de serem hipertensos, quando comparados aos seus pares ativos.

Além disso, no presente estudo, a obesidade e o sobrepeso fizeram-se presentes em 5.4 e $11.8 \%$ dos adolescentes, respectivamente. Embora os valores sejam elevados, os resultados obtidos neste estudo foram inferiores aos 
encontrados nos estudos envolvendo adolescentes de Porto Alegre (24.8\%) e Botucatu (33\%) (Pierine et al., 2006; Suñé, Costa, Olinto, \& Pattussi, 2007). Contrariamente, foram superiores a diversos outros estudos realizados em cidades brasileiras, como é o caso de Florianópolis, $12.7 \%$ (Romanzini, 2006) e Campina Grande, 14.4\% (Carvalho et al., 2007). Estudos realizados em diferentes regiões brasileiras demonstram prevalências de sobrepeso e obesidade em adolescentes entre $10.8 \%$ a $33.8 \%$ (Brasil et al., 2007; Giugliano \& Melo 2004; Oliveira et al., 2004). Ao comparar com estudos desenvolvidos com populações internacionais, a prevalência encontrada se mostrou superior em adolescentes sul-americanos (14.1\%) e europeus (11.1\%) (Paterno, 2003; Pileggi et al., 2005). Ressalta-se, portanto, que tanto nos estudos com amostra brasileira quanto nos internacionais, a ocorrência de excesso de peso é um fator presente.

A chance de os adolescentes obesos serem hipertensos foi 5.1 vezes maior, ao comparar com adolescentes normotensos, resultados esses que corroboram alguns estudos recentes, que evidenciaram uma prevalência de HA três vezes maior entre os adolescentes com sobrepeso e obesidade (Moura et al., 2004; Sorof et al., 2004). Em adolescentes argentinos, Paterno (2003) observou que a HA está significantemente associada ao sobrepeso ( $\mathrm{OR}=$ 2.9; IC95\% $=2.16-3.60)$ e à obesidade (OR $=4.9 ;$ IC95\% $=3.07-7.90$ ), associações essas que corroboram os resultados do atual estudo, ao apresentarem valores crescentes de risco para a associação entre o excesso de peso corporal e PA. Ainda em conformidade com os resultados acima descritos, o excesso de peso mantém-se atrelado a níveis comprometedores de PA. Brasil (2008) afirma que a inatividade física é um dos fatores que influencia no surgimento do sobrepeso e da obesidade, conforme podemos observar nos resultados apresentados na tabela 3.

Observou-se que os adolescentes inativos e os insuficientemente ativos apresentaram 7.97 e 4.35 vezes mais chances de adquirir excesso de peso corporal comparados aos adolescentes ativos. Mesmo apresentando valores inversos ao do atual estudo, Jenovesi, Bracco, Colugnati, e Taddei (2003) demonstraram, em um estudo realizado em São Paulo, que as chances de os adolescentes com excesso de peso serem inativos eram duas vezes maiores do que aquelas apresentadas pelos seus pares eutróficos. Em outro estudo, desenvolvido na cidade de Niterói, Silva e Malina (2000), demonstraram que há uma duplicação das chances de um adolescente com comportamento sedentário desenvolver excesso de peso, em relação a um eutrófico. Janssen et al. (2005) fortalecem esta ideia ao evidenciarem que, em uma análise feita com adolescentes de 34 países, o aumento da AF reduziu as chances de os adolescentes de 29 países terem excesso de peso corporal. Vale reforçar que, para os adolescentes de Taguatinga, essas chances foram ainda mais elevadas.

A inatividade física e a alimentação inadequada podem levar a obesidade, hipertensão, podendo até mesmo evoluir para um quadro de síndrome metabólica, sendo o exercício físico um importante fator para prevenção e tratamento desta síndrome (Ciolaq \& Guimarães, 2004). De uma maneira geral, a relação verificada entre o IMC, o NAF e a PA nos adolescentes investigados, sinaliza a AF como um componente importante nos programas de prevenção ou tratamento do excesso de peso e da HA. No entanto, extrapolar os resultados deste estudo com os de outras populações é uma tarefa complexa. Assim, algumas limitações devem ser apontadas, em primeiro lugar, alguns fatores como as variações de hábitos alimentares ou de estilos de vida não foram controlados. Além disso, a população do presente estudo foi limitada a adolescentes saudáveis, portanto, não pode ser considerada representativa de uma população normal. Outra limitação é a não análise de variáveis comportamentais e características sócio-econômicas. 


\section{CONCLUSÕES}

$\mathrm{Na}$ amostra estudada, o excesso de peso e o baixo NAF mostraram associação positiva com o desenvolvimento de HA. Além disso, baixo NAF esteve associado ao desenvolvimento de sobrepeso e obesidade nos adolescentes. Assim, os dados deste estudo reforçam a necessidade de intervenções voltadas à redução dos níveis pressóricos e do sobrepeso e da obesidade, por meio do incremento da prática de atividade física como fator de prevenção e tratamento não medicamentoso da HA.

Agradecimentos:

Nada declarado.

\section{Conflito de Interesses:}

Nada declarado.

Financiamento:

Nada declarado.

\section{REFERÊNCIAS}

Ainsworth, B. E., Haskell, W. L., Whitt, M. C., Irwin, M. L., Swartz, A. M., Strath, S. J.,...Leon, A. S. (2000). Compendium of physical activities: An update of activity codes and met intensities. Medicine and Science in Sports and Exercise, 32(9 suppl), S498-S504.

Araújo, T. L., Lopes, M. V. O., Cavalcante, T. F., Guedes, N. G., Moreira, R. P., Chaves, E. S., \& Silva, V. M. (2008). Análise de indicadores de risco para hipertensão arterial em crianças e adolescentes. Revista da Escola de Enfermagem da USP, 42(1), 120-126. doi: 10.1590/S008062342008000100016.

Associação Brasileira para o Estudo da Obesidade e Síndrome Metabólica - ABESO (2008). Dia do combate ao sedentarismo. Retirado em 15 de maio de 2011, a partir de http://www.abeso.org.br/ reportagens/dia_nacional_sedentarismo.htm

Azevedo, M. R., Araújo, C. L., Silva, M. C., \& Hallal, P. C. (2007). Tracking of physical activity from adolescence to adulthood: a population-based study. Revista de Saúde Pública, 41 (1), 69-75. doi: 10.1590/S0034-89102007000100010.

Barros, M. V. G., \& Nahas, M. V. (2003). Medidas da atividade física: Teoria e aplicação em diversos grupos populacionais. Londrina: Midiograf.

Brasil, Ministério da Saúde. Secretaria de Vigilância em Saúde. Secretaria de Atenção à Saúde. Instituto Nacional do Câncer. Coordenação de Prevenção e Vigilância (2004). Inquérito Domiciliar sobre Comportamentos de Risco e Morbidade Referida de Doenças e Agravos Não-Transmissíveis: Brasil. 15 capitais e Distrito Federal, 2002-2003. Rio de Janeiro: INCA.

Brasil. Ministério da Saúde, Secretaria de Atenção a Saúde, Departamento de Atenção Básica (2008). Guia alimentar para a população Brasileira. Promovendo a alimentação saudável/Ministério da saúde, Secretaria de atenção a saúde, coordenação geral da política de alimentação e nutrição. Série A. Normas e manuais técnicos. Brasília: Ministério da Saúde.

Brasil, L. M. P., Fisberg M., \& Maranhão, H. S. (2007). Excesso de peso de escolares em região do Nordeste Brasileiro: contraste entre as redes de ensino pública e privada. Revista Brasileira de Saúde Materno-Infantil, 7(4), 405-412. doi: 10.1590/S1519-38292007000400008.

Carvalho, D. F., Paiva, A. A., Melo, A. S. O., Ramos, A. T., Medeiros, J. S., Medeiros, C. C. M., \& Cardoso, M. A. A. (2007). Perfil lipídico e estado nutricional de adolescentes. Revista Brasileira de Epidemiologia, 10(4), 491-498. doi: 10.1590/S1415-790X2007000400007.

Cole, T. J., Bellizzi, M. C., Flegal, K. M., \& Dietz, W. H. (2000). Establishing a standard definition for child overweight and obesity worldwide: international survey. British Medical Journal, 320, 1240-1243. doi: 10.1136/bmj.320.7244.1240.

Chiara, V., Sichieri, R., \& Martins, P. D. (2003). Sensibilidade e especificidade de classificação de sobrepeso em adolescentes, Rio de Janeiro. Revista de Saúde Pública, 2(37), 226-231. doi: 10.1590/S0034-89102003000200010.

Ciolaq, E. G., \& Guimarães, G. V. (2004). Exercício físico e síndrome metabólica. Revista Brasi- 
leira de Medicina do Esporte, 10(4), 319-324. doi: 10.1590/S1517-86922004000400009.

Farias, E. S., \& Salvador, M. R. D. (2005). Antropometria, composição corporal e atividade física de escolares. Revista Brasileira de Cineantropometria e Desempenho Humano, 7(1), 21-29.

Field, A. (2009). Descobrindo a estatística usando o SPSS. Porto Alegre: Artmed.

Giugliano, R., \& Melo, A. L. P. (2004). Diagnóstico de sobrepeso e obesidade em escolares: Utilização do índice de massa corporal segundo padrão internacional. Jornal de Pediatria, 80(2), 129-134. doi: 10.1590/S0021-75572004000200010.

Guedes, D. P., Guedes, J. E. R. P., Barbosa, D. S., \& Oliveira, J. A. (2006). Fatores de risco cardiovasculares em adolescentes: indicadores biológicos e comportamentais. Arquivos Brasileiros de Cardiologia, 86(6), 439-450. doi: 10.1590/ S0066-782X2006000600006.

Guerra, S., Ribeiro J., Duarte J., \& Mota J. (2003). Obesidade e Hipertensão Arterial em Idades Pediátricas na Área do Grande Porto. Acta de Pediatria, 34(6), 395-400.

Hedley, A. A., Ogden, C. L., Johnson, C. L., Carroll, M. D., Curtin, L. R., \& Flegal, K. M. (2004). Prevalence of overweight and obesity among us children, adolescents, and adults, 19992002. The Journal of the American Medical Association, 23(291), 2847-2850. doi: 10.1001/ jama.291.23.2847.

Jardim, N., Povoa, R., Fo, B. L., Cavichio, L., Costa, E., Ferreira, C.,...Carvalho, A. C. C. (2011). Prevalence of hypertension in adolescents of the Brazilian Amazonic Region. American Journal of Hypertension, 14, S191A. doi:10.1016/S08957061(01)01691-0.

Jenovesi, J. J., Bracco, M. M., Colugnati, F. A. B., \& Taddei, J. A. A. C. (2003). Perfil de atividade física em escolares da rede pública de diferentes estados nutricionais. Revista Brasileira de Ciência e Movimento, 11 (4), 57-62.

Janssen, I., Katzmarzyk, P. T., Boyce, W. F., Vereecken, C., Mulvihill, C., Roberts, C.,...Pickett, W. (2005). Comparison of overweight and obesity prevalence in school-aged youth from 34 coun- tries and their relationships with physical activity and dietary patterns. The International Association for the Study of Obesity. Obesity reviews, 6(2), 123-132. doi: 10.1111/j.1467789X.2005.00176.x.

Kearney, P. M., Whelton, M., Reynolds, K., Muntner, P., Whelton, P. K., \& He, J. (2005). Global burden of hypertension: analysis of worldwide data. Lancet, 365(9455), 217-223 doi:10.1016/ S0140-6736(05)17741-1.

Lohman, T. G., Roche, A. F., \& Martorell R. (1988). Anthropometric standardization reference manual. Champaing, IL: Human Kinetics Books.

Luiz, R. R., \& Magnanini, M. M. F. (2000). A lógica da determinação do tamanho da amostra em investigações epidemiológicas. Cadernos de Saúde Coletiva, 8(2), 9-28.

Monge, R., \& Beita, O. (2000). Prevalence of coronary heart disease risk factors in Costa Rican adolescents. Journal of Adolescent Health, 27(3), 210-217. doi:10.1016/S1054139X(99)00017-8.

Moura, A. A., Silva, M A. M., Ferraz, M. R. M. T., \& Rivera, I. R. (2004). Prevalência de pressão arterial elevada em escolares de Maceió. Jornal de Pediatria, 80(1), 35-40. doi: 10.1590/S002175572004000100008.

National High Blood Pressure Education Program Working Group on High Blood Pressure in Children and Adolescents (2004).The fourth report on the diagnosis, evaluation, and treatment of high blood pressure in children and adolescents. Pediatrics, 114(suppl2), 555-576.

Oliveira, A. M. A., Oliveira, A. C., Almeida, M. S., Almeida, F. S., Ferreira, J. B. C., Silva, C. E. P., \& Adan, L. F. (2004). Fatores ambientais e antropométricos associados à hipertensão arterial infantil. Arquivos Brasileiros de Endocrinologia Metabologia, 48(6), 849-854. doi: 10.1590/ S0004-27302004000600011.

Oliveira, R. G., Lamounier, J. A., Oliveira, A. D. B., Castro, M. D. R., \& Oliveira, J. S. (1999). Pressão arterial em escolares e adolescentes o estudo de Belo Horizonte. Jornal de Pediatria, $75(4), 256-266$. 
Paradis, G., Lambert, M., O’Loughlin, J., Lavallée, C., Aubin, J., Delvin, E.,...Hanley, J. A. (2004). Blood pressure and adiposity in children and adolescents. Circulation, 110(13), 1832-1838. doi: 10.1161/01.CIR.0000143100.31752.B7.

Paterno, C. A. (2003). Coronary risk factors in adolescence. The Fricela Study. Revista Española de Cardiologia, 56(5), 452-458.

Paulus, D., Saint-Remy, A., \& Jeanjean, M. (1999). Blood pressure during adolescence: a study among Belgian adolescents selected from a high cardiovascular risk population. European Journal of Epidemiology, 15(9), 783-790. doi: 10.1023/A:1007670613848.

Pierine, D. T., Carrascosa, A. P. M., Fornazari, A. C., Watanabe, M. T., Catalani, M. C. T., Fukuju, M. M.,...Maestá, N. (2006). Composição corporal, atividade física e consumo alimentar de alunos do ensino fundamental e médio. Motriz, 12(2) 113-124.

Pileggi, C., Carvone, V., Nobile, C. G. A., \& Pavia, M. (2005). Blood pressure and related cardiovascular disease risk factors in 6-18 year-old students in Italy. Journal of pediatrics and Child Health, 41(7), 347-352.doi: 10.1111/j.14401754.2005.00629.x.

Romanzini M. (2006). Pressão arterial elevada em adolescentes: prevalência e fatores associados. (Dissertação de Mestrado, Universidade federal de Santa Catarina, 2006).

Silva, K. S., \& Farias Júnior, J. C. (2007). Fatores de risco associados à pressão arterial elevada em adolescentes. Revista Brasileira de Medicina do
Esporte, 13(4), 237-240. doi: 10.1590/S151786922007000400005.

Silva, K. S., Silva, F. M., \& Martins, C. M. L. (2006). Reprodutibilidade de um questionário de atividade física em crianças do município de João Pessoa, PB, Brasil. Revista Brasileira de Educação Física e Esporte, 20, 275.

Silva, R. C. R., \& Malina, R. M. (2000). Nível de atividade física em adolescentes do município de Niterói, Rio de Janeiro, Brasil. Cadernos de Saúde Pública, 16(4), 1091-1097. doi: 10.1590/ S0102-311X2000000400027.

Sociedade Brasileira de Cardiologia - SBC, Sociedade Brasileira de Hipertensão - SBH \& Sociedade Brasileira de Nefrologia - SBN (2004). IV Diretrizes Brasileiras de Hipertensão Arterial. Arquivos Brasileiros de Cardiologia, 82(supl. IV), $1-14$.

Sorof, J. M., Lai, D., Turner, J., Poffenbarger, T., \& Portman, R. J. (2004). Children overweight, ethnicity, and the prevalence of hypertension in school-aged. Pediatrics, 113(3), 475-482.

Suñé, F. R., Dias-da-Costa, J. S., Olinto, M. T. A., \& Pattussi, M. P. (2007). Prevalência e fatores associados para sobrepeso e obesidade em escolares de uma cidade no Sul do Brasil. Cadernos de Saúde Pública, 23(6), 1361-1371. doi: 10.1590/ S0102-311X2007000600011.

World Health Organization (WHO) - (2003). Global strategyon diet, physical activity and health. Obesity and overweight. Retirado em 15 de maio de 2011, A partir de http://www.who.int/hpr/ NPH/docs/gs_obesity.pdf

(cc) EY-No Todo o conteúdo da revista Motricidade está licenciado sob a Creative Commons, exceto quando especificado em contrário e nos conteúdos retirados de outras fontes bibliográficas. 